\title{
Digital Earth: A World Infrastructure for Sustaining Resilience in Complex Pandemic Scenarios
}

\author{
Richard Simpson
}

This perspective makes the case for an improved multidisciplinary global collaboration that more effectively leverages our technological prowess to improve how we share and communicate the best science-based information. The concept of Digital Earth - the aspirational digital representation of our physical, social, and natural world - is introduced to serve as a critical digital infrastructure for proactively informing policy, investment, planning, design, and our behavioural responses to managing crisis situations, including pandemics.

\subsection{Spatial Information During a Pandemic}

We live in an intricately interconnected and increasingly globalised world. Within hours a viral infection can rapidly spread throughout a community. Despite the advances in sciences and technologies, a pandemic such as COVID-19 can cost the global economy many trillions of dollars and have far reaching global social, economic, and political consequences.

Spatial information has long been recognised as a critical enabler in the forensic management of infectious diseases ever since 1854 when Dr. John Snow, one of the founders of modern epidemiology, applied maps to trace the source of cholera outbreaks to a single contaminated pump in Broad St. Soho, London [1].

During the COVID-19 pandemic there has been a plethora of innovative applications using spatial information to resolve issues at specific points in the response, and also recovery phases. These location-enabled apps have in many instances been helpful to specific tasks such as contract tracing, drug delivery and presenting signage via drones and robots, finding ventilators, dashboards, spatial enablement of e-learning and e-health systems, finding availability and location of public services, and informing us on lock-down policies.

However, despite the benefits of impressive technological and scientific advances, we are still effectively applying maps as Snow did over one and half centuries ago, except now the maps are digital. With uncoordinated international cohesion; siloing of information across disciplines, organisations and jurisdictions; and a lack of trusted integrated information resources, we have seen a cargo cult emerge with Government organisations practicing rituals to win public and political favour as hero warriors in this 'war' on the virus. We are not facing up to these challenges and their multi-dimensional dynamics as emergent complex adaptive systems, instead we are too often simplifying problems down into the arbitrary domains convenient to focus mono-disciplinary application of commercial-off-the-shelf point 'solution' technologies. We also lack adequate regulations, policies and data mandates to support the governance and sustained use, 
provisioning and sharing of spatial information. The accumulative crisis now facing humanity and our inabilities to address it objectively is the result of failing to account for the aggregate impact of decisions initially made for short-term comforts and political gain.

New thinking is sought to revitalize these cultural and societal approaches towards spatial information and its metaphorical expression of objective science and our own identity and associations with places. This renaissance culminating in the realisations of a Digital Earth will expand our horizons for the interpretation of events, and create new ways for expressing our situational awareness through the arts, philosophy and scientific inquiry.

\subsection{A New Paradigm of Thinking}

To advance beyond this stalemate we need to shift the paradigm and adopt a more holistic and deterministic perspective on the challenge. We need to consider spatial information as an integrating enabler for a common open and innovative digital infrastructure, not just as a location enabler for point applications.

The rapid urbanisation, easy mobility and dense interconnectivity of cities is creating environments where pandemics will thrive. There is irony in the fact that the built and social environments of our city centric civilisation has made us more vulnerable to severe impact from disasters. Unbridled complexity of our built environment exposes our cities to risks of catastrophic failure. We have seen this demonstrated repeatedly in recent history with floods, earthquakes, bushfires, wars, tsunamis, landslides, and cyclones. The intensity of these disasters is increasing in the wake of our changing climate, and accelerating competition for essential resources.

Disasters can happen concurrently, as demonstrated by the spread of cholera following the Haiti Earthquake in 2010 [2]. The Spanish flu of 1918 brought acute illness to over a quarter of the world's population and death to an estimated 40 million people in the aftermath of 10 million killed in World War One.

The likelihood for disasters to happen concurrently is now more probable with the changing climate and city-driven global economy in the wake of rapid urbanisation. The significant human impact on the Earth's ecosystems and climate change increases the risk as this is creating situations more conducive to breeding pandemics [2].

Snow's identification of a contaminated water pump illustrates how even the infrastructure we depend upon and trust or fresh water and sanitation in a city can fail with disastrous consequence.

Despite so many advances in asset information management, for the greater part maintenance practices still remain reactive - just as they would have been in Snow's time. There is a pressing need for our cities' utilities to transform their business processes, policies and mandates to shift their traditional practices to a paradigm where there is massive integration of trusted evidence-based spatial data with real-time cyber-physical systems. Through this more dynamic ability to digitally mirror the objects and processes of the physical world such organisations and their supply chains can improve resilience with the applications of $\mathrm{AI}$ and machine learning with more proactive predictive and prescriptive asset management [3].

Similarly, decision making must also be driven by scientific evidence and effective use of spatial information to more effectively meet the challenges of pandemics such as COVID-19. Government and business institutions have traditionally been enabled by technical data and information but the power structures from twentieth century mean decisions are often made for short term vested interests rather than public good. Recent wide access to emerging communication technologies and social media platforms have revealed an emerging public capability for use and sharing of expert data in more productive ways to inform human behaviour for survival.

This pandemic has demonstrated that effective responses demands transparent governance and collaboration between all tiers of Government, related agencies and civilians. Snow's achievement not only illustrates the power of maps in the visualisation of correlated data, but also how spatial indexing helps build evidence with veracity and provides a referencing system for the aggregation of the collated information. Any disaster, especially a pandemic demands a coordinated 
real-time response from diverse interdisciplinary teams that may include scientists, politicians, health workers, supply chain operators, data scientists, foreign diplomats, and technologists.

It therefore helps if all these stakeholders have access to the same trusted spatially referenced data. For population wide implementation of an emergency response, such data needs to be communicated clearly and accurately to the civilian population in ways that engage their cooperation and inform their personal choices to change habitual behaviours.

\subsection{Digital Earth}

Digital Earth is a multidisciplinary collaboration to build a comprehensive digital twin of our planet's built, natural and social environments [4]. It is envisioned as a massively integrated multi-disciplined digital representation of our knowledge expressed through spatial metaphor of a globe. For example, a Digital Earth could spatially integrate our planet's thermodynamic performances with associated environmental, epidemiological, economic, and other social phenomena so we can more effectively monitor situations and inform better decisions during all phases of a disaster. Digital Twins are the building blocks of a Digital Earth. They integrate multi-dimensional geometric, topological, behavioural and semantic representations with Internet of Things (IoT), artificial intelligence (AI) and analytics. Graph based digital threads trace all lifecycle transactions and associations to ensure the integrity of this cyber-physical coupling [3]. Through metaphors such as virtual globes [5] we can apply this digital framework to build a deeper and more collaborative understanding of the complex dynamic interactions in our physical world and what bearing these may have on our individual and collective wellbeing.

As a global scientific project, Digital Earth sits on a seamless multi-scaled continuum with other big-science initiatives including the Physiome project [6] to model our physiology for drug discovery and testing, and its predecessor the Human Genome project to functional and physically map our genes. These mathematical models provide insight into the dynamic complex adaptive systems that we have evolved into and become part of.

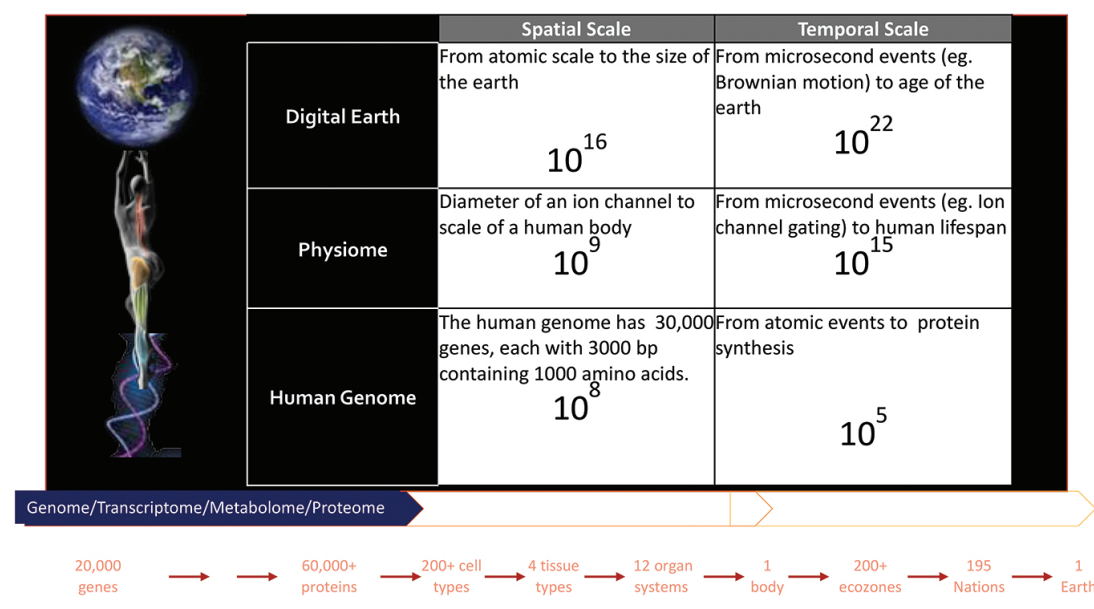

\section{FIGURE 32.1}

Big Science continuum - Adapted from Hunter, PJ and Borg, TK. Integration from proteins to organs: The Physiome Project. Nature Reviews Molec \&3 Cell Biol. 4:237-243, 2003

For example, the models could reveal the interaction between COVID-19 the environment and physiological functions at different scales from genes to the whole living organism to the environmental factors. At the scale of the Physiome, genome and other initiatives in this continuum 
such as the metabolome and transcriptome [6] valuable insights are being sought to accelerate the quest for a vaccine and improve patient treatment. Similarly, at the next magnitude in scale there is exciting potential for Digital Earth to provide predictive early warnings and deliver trusted evidence-based prescriptive course of action in real-time at every phase and inter-phase transition of a multi-hazard crisis situation.

A Digital Earth could also serve as an integrating foundation for a next generation of digital passports to enable us to safely travel and serve as an augmented super-sense to protect us from harm. This will only be possible if the Scientific and technical developments underpinning this project are effectively shared with, questioned, and trusted by civilian populations so they become a part of the civil and political process of learning and reconceptualising our impact on our shared global habitat and the laws for our engagement with each other.

\subsection{Conclusion}

There are remedial lessons to learn from this pandemic about appreciating how we are all connected, and how technologies can shape our daily existences and our survival outcomes. In the past we have embraced a vision of a world of machines for living in and enabling our mobility on demand. Until now we have only superficially applied technologies to conveniently interpret the realities of the world as a simple metaphor. Even with the benefit of computer technologies we model this world rather simplistically with a domain-based point 'solution' approach, avoiding complexity of the multi-disciplinary and massive data integration. This has so often given us a mistaken mental picture of possibilities and led us astray. There is now some urgency to rethink the design of our habitats, make scientifically informed decisions, transparently communicate trusted information and to consider how we can cyber-physically engage in the flux of the emergent complex adaptive systems of the world we live in rather than the one we picture.

A Digital Earth is a critical digital infrastructure to ensure our future survival as it consolidates our collective knowledge and liberates new wisdom. Realising a Digital Earth will demand prioritisation, fresh new 'moon-shot' thinking and international collaboration and determination. There are many intellectual, ethical and philosophical challenges, but a Digital Earth may become the most significant scientific and technological achievement in our civilised history and the definitive achievement of the $21^{\text {st }}$ Century.

\section{References}

[1] Tom Koch. Cholera, chloroform, and the science of medicine: A life of john snow. Cartographic Perspectives, 60:62-64, 06 2004. doi: 10.14714/CP48.461.

[2] Bapon Fakhruddin, Kevin Blanchard, and Durga Ragupathy. Are we there yet? The transition from response to recovery for the COVID-19 pandemic. Progress in Disaster Science, 7:100102, 2020. ISSN 2590-0617. doi: https://doi.org/10.1016/j.pdisas.2020.100102.

[3] D Jackson and R Simpson. Digital City Manual for Digital Earth part 3 chapter 15. Springer, New York, 2020. URL https://link.springer.com/content/pdf/10.1007\%2F978-981-32-9915-3_16.pdf.

[4] Michael F Goodchild, Huadong Guo, Alessandro Annoni, Ling Bian, Kees De Bie, Frederick Campbell, Max Craglia, Manfred Ehlers, John Van Genderen, Davina Jackson, et al. Next-generation digital earth. Proceedings of the National Academy of Sciences U S A, 109(28):11088-11094, 2012. doi: 10.1073/pnas.1202383109.

[5] Albert Gore. Earth in the balance-ecology and the human spirit. Houghton Mifflin Harcourt, New York, 1992.

[6] Peter J. Hunter and Thomas K. Borg. Integration from proteins to organs: the physiome project. Nature Reviews Molecular Cell Biology, 4(3):237-243, Mar 2003. ISSN 1471-0080. doi: 10.1038/nrm1054. 